\title{
Chimeric antigen receptors for the adoptive $T$ cell therapy of hematologic malignancies
}

\author{
Marco L. Davila • Diana C. G. Bouhassira • \\ Jae H. Park · Kevin J. Curran · Eric L. Smith • \\ Hollie J. Pegram $\cdot$ Renier Brentjens
}

Received: 5 October 2013/Accepted: 14 November 2013/Published online: 6 December 2013

(C) The Japanese Society of Hematology 2013

\begin{abstract}
The genetic modification of autologous T cells with chimeric antigen receptors (CARs) represents a breakthrough for gene engineering as a cancer therapy for hematologic malignancies. By targeting the CD19 antigen, we have demonstrated robust and rapid anti-leukemia activity in patients with heavily pre-treated and chemotherapy-refractory B cell acute lymphoblastic leukemia (BALL). We demonstrated rapid induction of deep molecular remissions in adults, which has been recently confirmed in a case report involving a child with B-ALL. In contrast to the results when treating B-ALL, outcomes have been more modest in patients with chronic lymphocytic leukemia (CLL) or other non-hodgkin's lymphoma (NHL). We review the clinical trial experience targeting B-ALL and CLL and speculate on the possible reasons for the different outcomes and propose potential optimization to CAR T cell therapy when targeting CLL or other indolent NHL. Lastly, we discuss the pre-clinical development and potential for clinical translation for using CAR $\mathrm{T}$ cells against multiple myeloma and acute myeloid leukemia. We highlight the
\end{abstract}

M. L. Davila · J. H. Park · E. L. Smith .

H. J. Pegram · R. Brentjens $(\square)$

Leukemia Service, Department of Medicine, Memorial

Sloan-Kettering Cancer Center, 1275 York Avenue,

New York, NY 10065, USA

e-mail: brentjer@mskcc.org

M. L. Davila

e-mail: davilam@mskcc.org

D. C. G. Bouhassira

Center for Cell Engineering, Sloan-Kettering Institute, Memorial

Sloan-Kettering Cancer Center, New York, NY, USA

K. J. Curran

Department of Pediatrics, Memorial Sloan-Kettering Cancer

Center, New York, NY, USA potential risks and benefits by targeting these poor outcome hematologic malignancies.

Keywords CAR - Hematologic malignancies . Adoptive $\mathrm{T}$ cell therapy

\section{Introduction}

Gene therapy has demonstrated significant potential as a cancer therapy in the last few years. The greatest successes have been reached by genetic modification of autologous patient $\mathrm{T}$ cells with chimeric antigen receptors (CARs), which are novel and synthetic receptors composed of the antigen-binding domain from a B cell receptor fused to the signaling elements associated with a $\mathrm{T}$ cell receptor [1]. Genetic modification of a $\mathrm{T}$ cell with a CAR successfully re-directs the $\mathrm{T}$ cell towards the target of the CAR. Ligation of the CAR results in signal transduction through signaling moieties and leads to activation of the $\mathrm{T}$ cell and killing of the target directly or through engagement of other components of the immune system (Fig. 1). The synthetic nature of CARs allows for the targeting of a variety of cancers by simply substituting various antigen-binding domains, encoded by single chain variable fragments $(\mathrm{scFv})$. Furthermore, selection of different signaling moieties could potentially support different $\mathrm{T}$ cell functions or properties. More detailed reviews on the implications of CAR design have been published $[2,3]$.

The translation of this therapy to the clinical setting has required the development of a robust, rapid, and reproducible method of producing CAR T cells (Fig. 2). Furthermore, while initial pre-clinical analyses of CARs involved various antigens and malignancies, most clinical trials have focused on B cell malignancies by targeting the 
Fig. 1 CAR T cell activation and killing of tumor targets. Tumor cell recognition occurs when a CAR on a $\mathrm{T}$ cell ligates its antigen on the tumor. Signaling and activation is mediated by the intracytoplasmic signaling domains within the CAR. Activation can lead to direct cytotoxicity of tumor target by CAR T cell mediated release of granzyme and perforin. Tumor killing can also be mediated by activation of other components of the immune system through release of cytokines by $\mathrm{CD} 4^{+} \mathrm{T}$ cells. Long-term eradication and prevention against tumor relapse may be provided by long-term memory CAR T cells that form after the initial activation
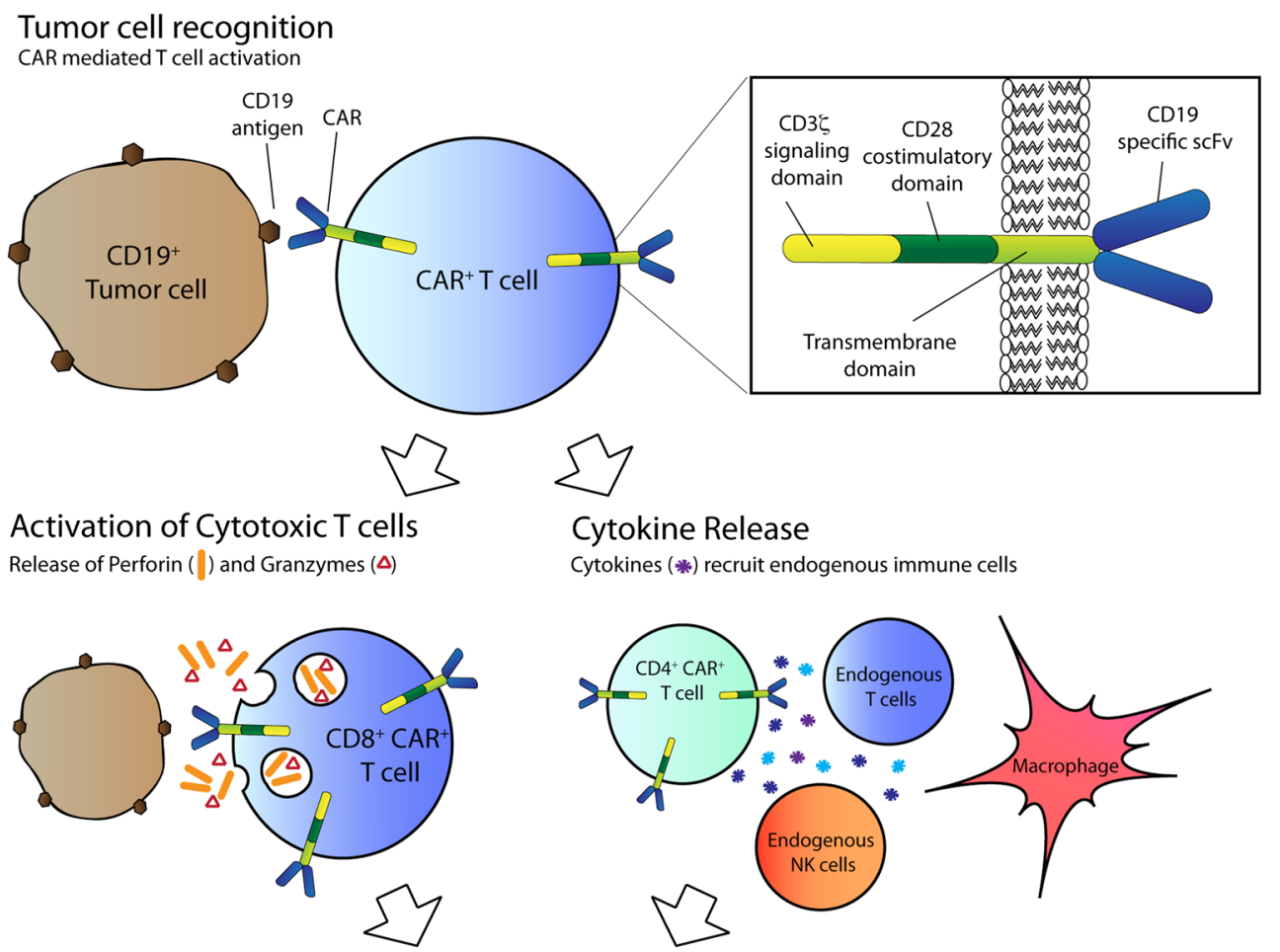

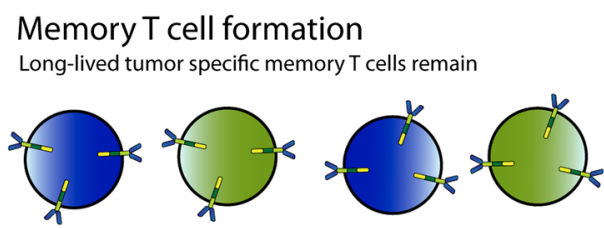

pan-B cell antigen, CD19. The first trials targeting CD19 involved patients with relapsed indolent non-Hodgkin's lymphomas (NHL) and chronic lymphocytic leukemia (CLL) demonstrating the safety of CAR $\mathrm{T}$ cells and occasional clinical benefit [4-10]. In contrast, two independent trials that infused CD19-targeted CAR T cells in patients with B cell acute lymphoblastic leukemia (B-ALL) resulted in robust anti-tumor efficacy and positive clinical outcomes, two feats not consistently reached with the trials involving NHL and CLL [11, 12]. Clearly, the proof-ofprinciple of gene therapy as a cancer therapy, in the form of CAR $T$ cells infused in patients with B-ALL, has been solidly demonstrated. Institutions, including our own at Memorial Sloan-Kettering Cancer Center (MSKCC), are currently seeking to extend these promising results in B-ALL to other hematologic malignancies. In this review, we will discuss clinical trials (Table 1) using CAR T cells in patients with NHL, CLL, and B-ALL, speculate on possible reasons for the different efficacies and outcomes observed in these trials, and suggest possible ways to enhance CAR T cell therapy for NHL. We will then discuss the state of development of CAR $\mathrm{T}$ cells for other hematologic malignancies in dire need of novel, cell-based gene therapies.

\section{B-ALL as a target for CD19 CAR T cells}

CD19 was chosen as a target for B cell malignancies because of its near-universal expression on $\mathrm{B}$ cell malignancies and its limited expression on B cells but not bone marrow (BM) stem cells [13, 14]. Since CD19 expression is specific to B cells and does not occur on other cells such as hematopoietic stem cells, no off-target effects would be expected when infusing patients with CD19 CAR T cells. Being one of the initial CAR systems developed, CD19 CARs became the first pre-clinical models used to establish key tenets of CAR T cell cancer therapy. For example, a critical limitation to CAR T cell therapy was noted with first-generation CARs, which are composed of a CD19targeted $\mathrm{scFv}$ and a $\mathrm{CD} 3 \zeta$ signaling component; these CARs had robust in vitro activity but limited pre-clinical in vivo efficacy. It was demonstrated that inclusion of a costimulatory receptor, such as CD28 or the $4-1 \mathrm{BB}$, with the $\mathrm{CD} 3 \zeta$ signaling protein in a second-generation CAR was sufficient to mediate robust killing of tumor targets in immunodeficient mouse models of human B cell malignancies [15-17]. Immunocompetent, syngeneic animal models were developed to determine how CAR $\mathrm{T}$ cells function in animals with abundant CD19 antigen expressed 


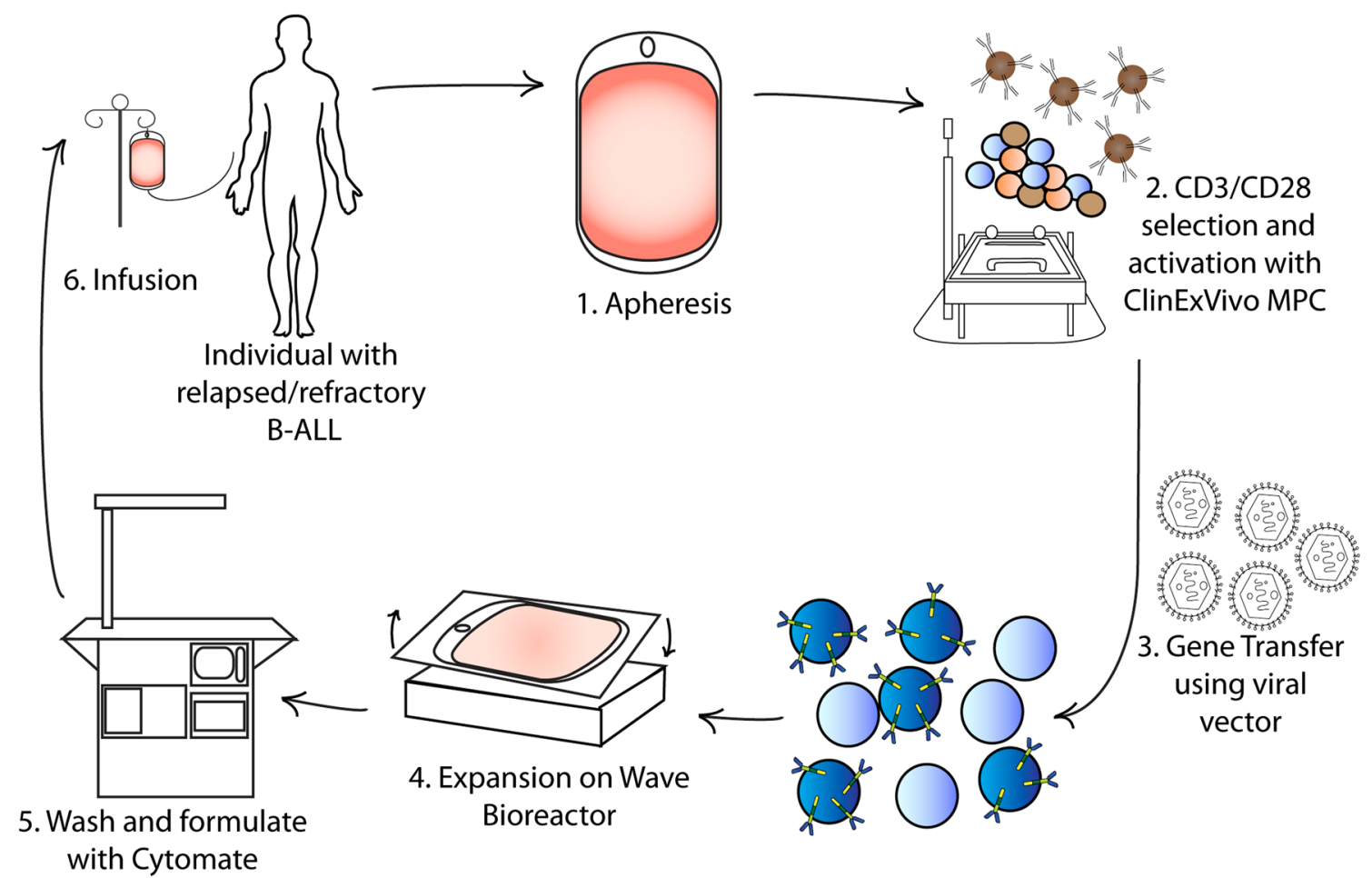

Fig. 2 Production of CAR T cells in a GMP facility. Production begins with (1) leukapheresis of the patient and is then followed by selection of $T$ cells (2), and their activation, from the leukapheresis product by positive selection with a CD3 antibody \pm a $\mathrm{CD} 28$

on normal B cells [18-21]. It was determined that for effective CD19 CAR T cell function some form of conditioning therapy was required, presumably in part to decrease the antigen burden or possible to deplete immunosuppressive regulatory $\mathrm{T}$ cells. Mice that were infused with CD19 CAR T cells without conditioning therapy showed no or only limited B cell killing but pre-treatment with a lymphodepleting conditioning agent resulted in B cell aplasia, tumor eradication, and long-term CAR $\mathrm{T}$ cell persistence. Lastly, one of the immunocompetent models validated B-ALL as a highly susceptible tumor target despite its aggressive and highly proliferative nature and also suggested that re-generating progenitor B cells in the $\mathrm{BM}$ could serve as an antigen reservoir to stimulate CD19 targeted CAR $\mathrm{T}$ cells and lead to long-term persistence [21]. The results from these pre-clinical studies served as the rationale for evaluating CD19 CAR T cells in humans with B cell malignancies (Table 1). They significantly influenced the design of these trials by focusing on CD19 CAR $\mathrm{T}$ cells modified with second-generation CARs, incorporating conditioning chemotherapy, and evaluating the therapy in B-ALL patients.

We opened the first Phase I clinical trial infusing CD19 CAR $\mathrm{T}$ cells into adults with relapsed/refractory B-ALL and the results were recently published [11]. Five patients antibody. After a few days the activated $\mathrm{T}$ cells are incubated with retroviral supernatant to transfer the CAR gene (3). Expansion, washing, and formulation result in infusion back into the patient (46). In general, at our center this process takes about 2 weeks

with chemotherapy-refractory B-ALL were enrolled and leukapheresed (Fig. 2). Despite extensive prior chemotherapy treatments and marked lymphopenia or elevated blast counts, we were able to collect a sufficient number of $\mathrm{T}$ cells, genetically target these cells to CD19, and subsequently expand the cells to the dose required by our clinical protocol $\left(3 \times 10^{6} \mathrm{CAR} \mathrm{T}\right.$ cells $\left./ \mathrm{kg}\right)$. Prior to CAR T cell infusion, four of the patients had residual disease (ranging from minimal to gross residual) despite standard, highdose, multi-agent chemotherapy regimens. All four of these patients were successfully induced into a minimal residual disease-negative (MRD-) state. Furthermore, it is well known that the standard of care for patient with relapsed B-ALL is an allogeneic stem cell transplant (allo-SCT), which offers the only hope for a durable remission [22]. However, it is an unfortunate reality that most relapsed/ refractory B-ALL patients are unable to be induced into the requisite CR to undergo an allo-SCT [23]. Four of the five patients treated on our trial were able to undergo an alloSCT, the fifth having medical contraindications to an alloSCT, which argues for the potential of CD19 CAR T cell therapy as a potentially life-saving bridge to allo-SCT [11].

Toxicities were associated with the CD19 CAR T cell therapy [11]. Two of the patients experienced high-grade fevers persisting for several days and also had adverse 


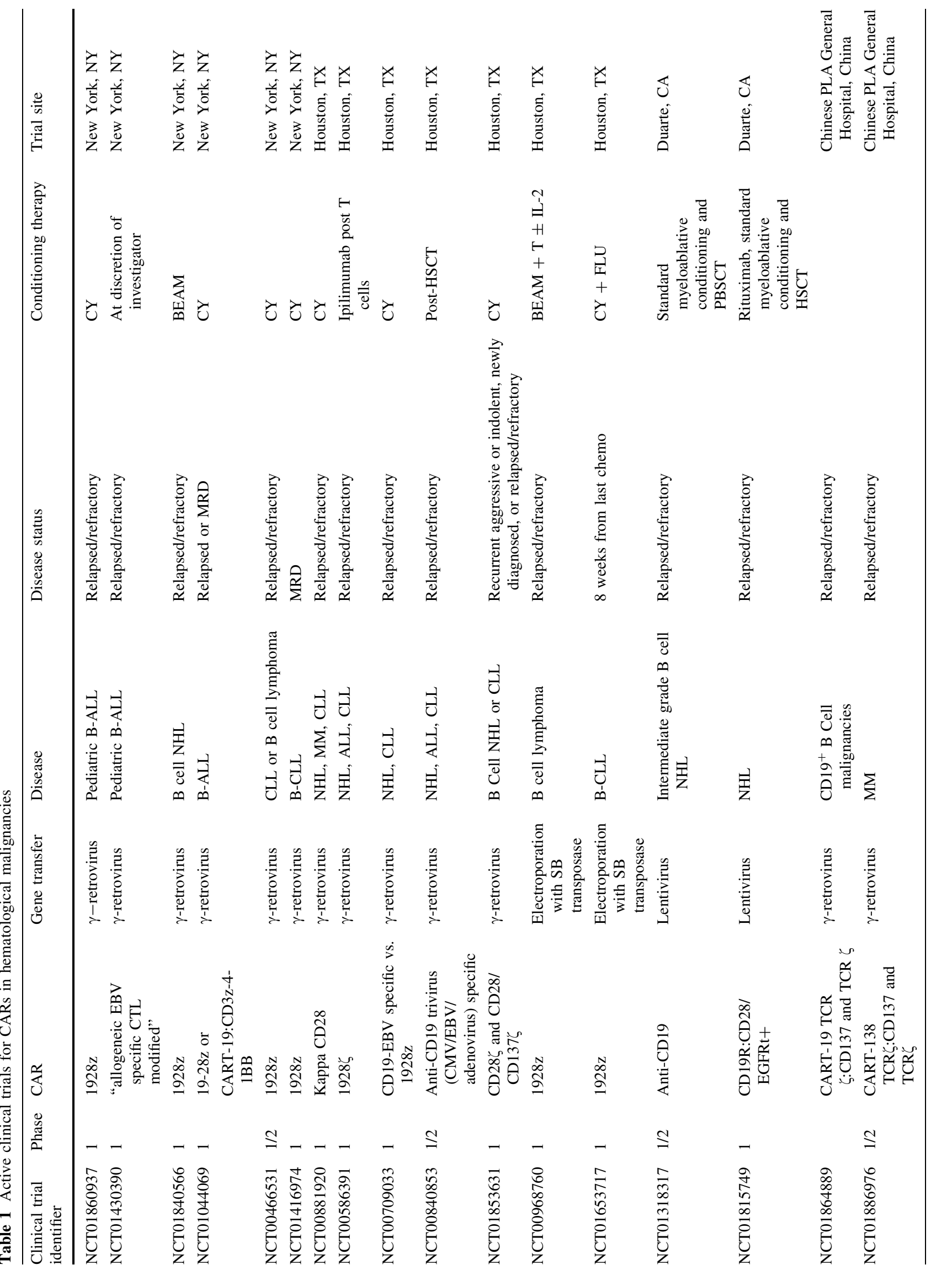




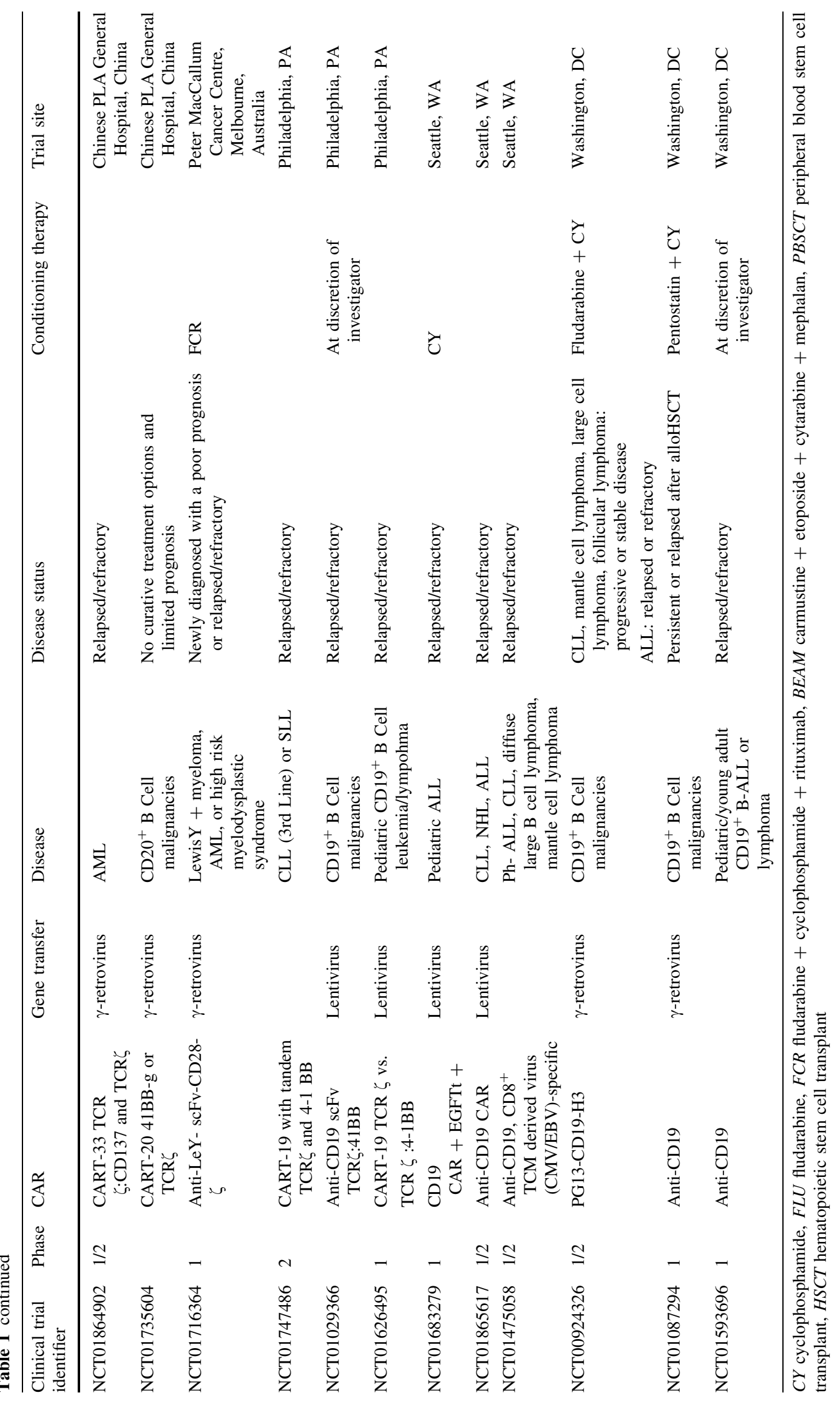


events including hypotension, obtundation, intubation, and seizures. These toxicities were manageable and also completely reversible with high-dose steroid administration. The other three patients experienced no major toxicities, although one patient had low-grade fevers. We determined that the severity of the toxicities was associated with a surge of inflammatory cytokines, suggesting that the toxicities were part of a cytokine release syndrome (CRS). Importantly, we made the novel observation that the extent of toxicities, as measured by cytokine surge, was strongly correlated with pre-CAR T cell infusion tumor burden [11]. Patients with high tumor burdens experienced a surge of cytokines and concomitant toxicities, while MRD+ patients experienced no or only mild cytokine increases and toxicities. However, regardless of the presence or lack of toxicities, all patients with residual disease were induced into a MRD- disease state [11].

These results have been recently confirmed by a case report of a pediatric B-ALL patient treated at the Children's Hospital of Pennsylvania (CHOP) [12]. This young patient had relapsed B-ALL and was infused with CD19 targeted CAR T cells. She experienced toxicities consistent with a CRS, which was ameliorated by anti-cytokine treatment with etanercept and tocilizumab. She was successfully induced into a MRD - remission, which to date has lasted for several months. A second patient described in this case report developed a morphologic remission but then had a rapid relapse of B-ALL tumor cells, which lacked the CD19 antigen [12]. Prior to CD19 CAR T cell infusion she had been treated with blinatumomab, which has been shown to induce CD19- B-ALL clones [24], so this may explain why these cells escaped targeting by the CD19 CAR T cells. This group also showed that CAR T cells traffic to the CNS, a location from which they could serve as tumor immunosurveillance or therapy since patients with B-ALL readily develop CNS disease [12].

While the outcomes of the two trials have been similar, there are a few important differences to consider [11, 12]. First of all, the second-generation CAR used in the CHOP trial utilized a 4-1BB signaling element compared to the CD28 signaling element used in our trial. This may account for some $\mathrm{T}$ cell physiologic differences such as onset and duration of CRS, $\mathrm{T}$ cell proliferation, and persistence. Furthermore, our trial used a gamma retrovirus to transfer the CD19 CAR gene, while the other trial used a lentiviral gene-transfer system, which is theoretically safer but has been less successful at consistently reaching stipulated CAR T cell dose [21]. Furthermore, while both trials involved relapsed B-ALL, one treated pediatric patients and the other treated adult patients. The disease is superficially the same, but the outcomes are dramatically different between adult and children: nearly all children with B-ALL are cured of their disease with chemotherapy alone and unfortunately most adults with B-ALL will eventually succumb to their disease.

Together, these two independent reports highlight the potential of CD19 gene-modified $\mathrm{T}$ cell therapy for a highly fatal and chemotherapy-refractory acute leukemia $[11,12]$. Important issues remain to be addressed such as a comparison of the efficacy of this therapy to standard salvage chemotherapy in a phase II trial. Furthermore, will these phase II trials be able to rapidly generate patientderived, gene-modified, cell therapies for a large number of patients at multiple treatment centers? Also, as more patients are treated with 41BB CAR $\mathrm{T}$ cells it will be important to find out if $\mathrm{B}$ cell aplasias present long-term complications that are not manageable with antibiotics and/ or intravenous immunoglobulins, as compared to patients treated with $28 \mathrm{z}$ CAR T cells who have normal B cell recovery after a few months of treatment. The two different CD19 CARs evaluated in B-ALL patients so far should also be compared to determine if they have significantly different efficacies or toxicities [11, 12]. Finally, another critical question to address is if the CD19 CAR T cell therapy is a stand-alone therapy or should only be used as a bridge to allo-SCT. Considering that allo-SCT is the current standard of care for relapsed B-ALL and that this is the only potential cure for adults, all patients eligible for an allo-SCT on our trials with reasonable morbidity and mortality risks will proceed to an allo-SCT [22, 23]. Even though these patients received an allo-SCT, we will still be able to detect anti-leukemia benefit with reasonable statistical power by comparing the standard relapse rate after allo-SCT (approximately $30 \%$ ) to the relapse rate of patients first treated with CD19 CAR T cell therapy.

\section{Targeting indolent $B$ cell malignancies with CD19 CAR T cells}

Investigators from the University of Pennsylvania (UPenn) initially reported durable complete remissions (CRs) in two patients and a partial remission (PR) in another patient who received autologous second-generation CD19-targeted CAR-modified $\mathrm{T}$ cells containing a 4-1BB co-stimulatory domain [8]. They presented an additional 6 patients at the 2012 American Society of Hematology (ASH) meeting, which included a CR in 1 patient, $P R$ in 3 patients but with a short follow-up ( $<5$ months), and treatment failures in 2 patients [25].

At our institution, we have published the outcomes of 8 patients with purine-analog refractory or relapsed CLL with bulky lymphadenopathy who received autologous CD19-targeted CAR-modified T cells containing a CD28 co-stimulatory domain. Of the 4 evaluable patients, one experienced a dramatic 
reduction of lymphadenopathy, and 2 patients had stable disease despite rapid tumor progression before therapy [10]. At the 2012 ASH meeting, we reported the clinical outcome of an additional 2 patients. One patient achieved a MRD-CR and another patient achieved a PR [26]. Investigators at the National Cancer Institute (NCI) reported the outcomes of 4 relapsed CLL patients treated with CD19-targeting CAR-modified autologous $\mathrm{T}$ cells containing CD28. Notably, all patients received nonmyeloablative conditioning therapy consisting of fludarabine and cyclophosphamide prior to $\mathrm{T}$ cell infusion. One patient achieved a CR, and 3 other patients achieved PRs [7].

The second-generation CARs used in these trials contain different co-stimulatory domains and they have not been compared directly, so it remains unclear whether any particular co-stimulatory molecule is superior to another in patients. Toxicities observed in these trials have been similar, mostly consisting of flu-like symptoms such as fever, malaise, and myalgias as well as capillary leak syndrome, likely related to a CRS. Prolonged B cell aplasia and hypogammaglobulinemia, anticipated side effects from on-target effects of the CD19-specific CAR-modified T cells, have also been observed in a subpopulation of responding patients. However, to date, there have been no apparent infectious complications attributed to genetically modified CD19 targeted T cells [27, 28].

Potential explanations for the limited clinical efficacy of CAR T cells in patients with CLL compared to B-ALL is the limited persistence of CAR $\mathrm{T}$ cells in patients with CLL, the immuno-inhibitory tumor microenvironment of CLL, the mostly bone marrow-based nature of B-ALL compared to the lymph-node based disease in CLL, and the lower tumor burden at treatment in patients with B-ALL. Potential methods to overcome these possible barriers include the incorporation of other signaling domains into the CAR systems or other immune effectors into the CAR $\mathrm{T}$ cells. By incorporating co-stimulatory domains such as CD28, CD137 (4-1BB), or CD134 (OX40) into third-generation CARs [29] or by directing secretion of proinflammatory cytokines such as IL12 [18] in a secondgeneration CAR, or "armored CAR", we may overcome limitations of persistence or a $\mathrm{T}$ cell inhibitory tumor microenvironment.

While early reports of autologous CD19-targeted CARmodified cells in patients with CLL or indolent NHL have been promising, obstacles remain. Carefully designed clinical trials will be needed to assess the optimal timing for incorporating CAR-based adoptive immunotherapies in CLL, and to test the safety and efficacy of the new thirdgeneration or armored CARs that may have enhanced activity against the chemotherapy-refractory bulky disease. These third-generation CARs will need to be equipped with an additional safety mechanism that will allow a quick elimination of the infused cells in cases of adverse events. One of the most well-studied strategies is to incorporate suicide genes, such as the herpes simplex thymidine kinase gene or an inducible caspase 9 (iCasp9) protein, that can be activated by specific drugs and eradicate the genetically engineered cells if adverse effects occur following adoptive transfer [30, 31]. Of the two suicide genes, iCasp9 seems to be the most appropriate for CAR-modified $\mathrm{T}$ cell therapy due to its rapid onset of action and relative lack of immunogenicity [32], and is currently being explored by several groups. Furthermore, one potential drawback of targeting CD19 or CD20 is the prolonged elimination of normal B lymphocytes and subsequent impairment of humoral immunity, although this can be mitigated with immunoglobulin repletion in patients. In order to reduce the toxicity to the normal B cells, CARs targeting CD23 [33], the $\kappa$ or $\lambda$ light chain of human immunoglobulin [34, 35], and ROR1 [36] are being explored. However, it remains to be seen whether these antigens will serve to be a safer and more effective targets for CAR-modified $\mathrm{T}$ cells in CLL and other indolent B cell NHL.

\section{Targeting multiple myeloma with CAR $T$ cells}

Multiple myeloma is a malignant disease of plasma cells that accumulate in the bone marrow. It is the second most common hematologic malignancy with 22,350 new cases expected to be diagnosed in the US in 2013 [37]. Myeloma is still generally considered an incurable disease outside an allo-SCT [37]. The median overall survival is considered $45-60$ months, although approximately $25 \%$ of patients present with high risk cytogenetics and have a median survival of less than 2 years [38].

Myeloma has several characteristics that make it an ideal disease to treat with adoptive $\mathrm{T}$ cell therapy. First, myeloma is primarily a disease of the bone marrow and adoptive $T$ cell therapy has been most successful in bone marrow predominant diseases such as ALL [11], when compared to extra-medullary diseases such as CLL [10]. Second, autologous stem cell transplantation is the standard of care in myeloma, and since lymphodepletion may enhance the efficacy of adoptive $\mathrm{T}$ cell therapy, the immediate post-autologous transplant period could be an optimal time for administering CAR T cells [10, 18]. Third, unlike all other treatments for myeloma, the graft vs. myeloma effect of an allo-SCT is potentially curative. Responses are seen even with minimal or no concomitant chemotherapy such as after a non-myeloablative transplant or after post-transplantation donor lymphocyte infusions [39]. However, while the graft vs. myeloma effect has great 
Table 2 Potential antigens, and off-targets, for a CAR T cell therapy against Multiple Myeloma

\begin{tabular}{ll}
\hline $\begin{array}{l}\text { CAR } \\
\text { target }\end{array}$ & Potential "on target, off tumor" cells recognized \\
\hline CD138 & $\begin{array}{c}\text { Bronchial epithelia [60] } \\
\text { CD38 }\end{array}$ \\
$\begin{array}{r}\text { Hematopoietic stem cells [60], NK cells [60], dendritic } \\
\text { cells [60], prostate [60], pancreas islet cells [61,62] }\end{array}$ \\
CD56 & CNS Neurons [60], NK cells [60] \\
\hline
\end{tabular}

potential, allo-SCTs are limited by transplant associated toxicity (including graft vs. host disease) and mortality, patient eligibility, and availability of a suitable donor. Thus, the discovery of a safer way to obtain the antitumor efficacy of the graft vs. myeloma response, such as CAR T cell therapy, is of great interest.

The most obvious targets for CAR therapy in myeloma are CD138, CD38, and CD56. In one study of over 300 patients with myeloma these antigens were expressed in 100,100 , and $78.7 \%$ of patient tumor samples, respectively [40]. CAR therapy targeting CD138 has entered into a phase I/II clinical trial in China this year (Table 1); however, to our knowledge there is no published preclinical or clinical data available for review. Pre-clinical data exist for CAR T cells targeting CD38 and CD56. Mihara et al. [41] have shown that second-generation CAR-modified T cells targeting CD38 are highly cytotoxic to CD38-positive human myeloma cell lines and primary myeloma cells isolated from human bone marrow in vitro. At MSKCC we have investigated second-generation CAR $\mathrm{T}$ cells targeted to CD56. These cells are highly cytotoxic to a CD56-positive human myeloma cell line both in vitro and in a systemic bone marrow predominant xenograft mouse model. In this model a dose of $5 \times 10^{6} \mathrm{CAR}$ T cells resulted in complete tumor regression [42]. However, unlike the CD19 antigen, these targets (CD138, CD38, CD56) are co-expressed on other important cell types and standard second-generation CAR therapy directed toward one of these molecules could potentially result in unacceptable on target, off tumor toxicity (Table 2). Therefore, researchers have investigated additional CAR targets. One potential strategy is second-generation CAR $\mathrm{T}$ cells targeted toward kappa light chain. These cells are cytotoxic against Ig-kappa positive tumor cell lines and primary CLL cells both in vitro and in vivo [35]. A phase I clinical trial using these cells is open to patients with myeloma and is underway at Baylor College of Medicine (Table 1). Similarly, there has been exciting pre-clinical results targeting the Lewis $\mathrm{Y}$ carbohydrate tumor antigen with CAR therapy in myeloma. In these studies, anti-Lewis Y CAR T cells delayed the growth of myeloma xenografts in NOD/SCID mice and improved their survival [43]. This observation has led to a phase I clinical trial, which opened in 2012 (Table 1).

Several other pre-clinical studies have been conducted with unique targets. The $\mathrm{B}$ cell maturation antigen (BCMA) has recently been targeted with a second-generation CAR in pre-clinical studies. These cells were shown to be cytotoxic to primary human myeloma cells in vitro and eradicated tumors and enhanced survival in a subdermal myeloma model in NSG mice [44]. A unique strategy for the use of CARs to target intracellular antigens in myeloma has also been developed; here, a single epitope of the intracellular tumor antigen NY-ESO-1 in the setting of HLA-A0201 serves as the CAR target. Using these CAR T cells in a systemic HLA-A0201 human myeloma cell line xenograft mouse model, tumor progression was delayed. Although the in vivo results were not as robust as the other pre-clinical data reviewed here, the concept potentially opens the door to targeting other intracellular tumor antigens [45]. While there is great promise for adoptive cell therapy in myeloma, clinical experience lags behind therapy for other B cell malignancies. Until we can assess the initial outcomes of current and future clinical trials we must wait to ascertain the promise of CAR T cell therapy for this distinct $\mathrm{B}$ cell malignancy.

\section{Targeting AML with CAR T cells}

Several pre-clinical reports have demonstrated that CAR-T cells have the potential to eradicate acute myelogenous leukemia (AML) [43, 46-48]. The first AML target to be successfully translated into a clinical trial has been the $\mathrm{Le}^{\mathrm{Y}}$ antigen [49]. In this report 4 patients with relapsed AML were treated using a CD28-containing second-generation CAR-targeting $\mathrm{Le}^{\mathrm{Y}}$. Patients received re-induction chemotherapy (fludarabine/cytarabine) and then CAR $\mathrm{T}$ cell infusion within 6 weeks of finishing chemotherapy [49]. Notably, one patient (\#4) with morphologic disease and leukemia cutis developed evidence of $\mathrm{T}$ cell activation following infusion. This included fever/rigors, reduction in peripheral blast count, and a transient skin rash, which on biopsy showed AML cells and CAR-T cells. Additional evidence supporting the efficacy of this target included a transient cytogenetic remission (patient \#5) and stable disease (patient \#2) for 23 months following infusion of CAR $\mathrm{T}$ cells. Detection of CAR T cells was also demonstrated for up to 4 months in both the peripheral blood and bone marrow following infusion. The most notable finding from this study may be the lack of toxicity following infusion despite the known expression of the $\mathrm{Le}^{\mathrm{Y}}$ antigen on normal tissues [50]. The implication is profound since $\mathrm{Le}^{\mathrm{Y}}$ is overexpressed on numerous epithelial cancers and therefore could become an important target for CAR T cells [50]. 
CAR T cells targeting CD33 or CD123 have also been the subject of pre-clinical reports demonstrating anti-leukemic potential against AML blasts [46-48]. CD33, which is overexpressed on the surface of $90 \%$ of AML blasts, is also the target of gemtuzumab ozogamicin (a humanized anti-CD33 monoclonal antibody combined with calicheamicin). Early enthusiasm for gemtuzumab was tempered by liver and hematologic toxicity related to accumulation of the conjugate calicheamicin and expression of CD33 on myeloid progenitors and Kuppfer cells [51]. However, the recent finding of improved survival in select patient populations has renewed interest in targeting CD33 [51]. One possible mechanism to improve the safety profile of CD33 targeting is through the use of CAR T cells thereby avoiding toxicity associated with calicheamicin. To this end, improved in vitro and in vivo anti-leukemic effect of cytokine-induced killer (CIK) cells and EBV-CTLs has been shown by transducing these cell types with an antiCD33 CAR [46, 47]. Importantly, these reports also assessed the impact that CD33 targeting would have on the normal CD33 expression myeloid compartment. Using in vitro assays, the targeting of normal hematopoietic progenitors expressing CD33 by anti-CD33 CAR transduced cells was demonstrated. However, despite this undesirable toxicity, residual clonogenic activity is maintained thereby indicating that it is a reversible toxicity $[46,47]$. To reduce the likelihood of hematopoietic toxicity, the targeting of the IL-3 receptor alpha subunit (CD123) has been tested. CD123 is widely overexpressed by AML blasts, is associated with apoptosis resistance, increased proliferation, poor prognosis and is limited in expression on hematopoietic progenitors [48]. CIK cells transduced with an anti-CD123 CAR have improved anti-leukemic effect while at the same time preserving hematopoietic stem cells in vitro [48]. Furthermore CIKs transduced with an anti-CD123 CAR have limited effect on normal monocytes and low-CD123 expressing endothelial cells [48]. Taken together, these results suggest that CARs targeting CD123 may have a lower toxicity profile with retained anti-leukemic effect when compared to anti-CD33 CARs. Regardless of which anti-AML CAR is ultimately advanced to clinical testing, the risk of unwanted on-target/off-tumor toxicity for both of these targets is high. Therefore, the first trials will require several safety mechanisms including dose escalation, dose splitting, and suicide genes (e.g., iCasp9) to safely test the efficacy of this approach.

\section{Conclusion}

The potential of CAR T cell therapy is evidenced by the dramatic successes observed in patients with relapsed, refractory B-ALL. Conflicting but encouraging results of this therapy in other hematological malignancies such as CLL, however, demonstrate the need for further optimization. Factors that require consideration when adapting the therapy to individuals with different hematological malignancies include chemotherapy conditioning regimens, CAR design, disease burden, and tumor target antigens. Continued follow-up with the patients that have participated in current clinical trials may provide further insight into whether this therapy best serves as a bridge to transplant or as a stand-alone cure. Overall, CAR T cell therapy stands out as an important novel therapeutic option for the treatment of B-ALL and one with significant potential for other hematologic malignancies.

Conflict of interest None.

\section{References}

1. Sadelain M, Riviere I, Brentjens R. Targeting tumours with genetically enhanced $\mathrm{T}$ lymphocytes. Nat Rev Cancer. 2003;3:35-45.

2. Davila ML, Brentjens R, Wang X, Riviere I, Sadelain M. How do CARs work?: early insights from recent clinical studies targeting CD19. Oncoimmunology. 2012;1:1577-83.

3. Sadelain M, Brentjens R, Riviere I. The basic principles of chimeric antigen receptor design. Cancer Discov. 2013;3:388-98.

4. Savoldo B, Ramos CA, Liu E, Mims MP, Keating MJ, Carrum G, et al. CD28 costimulation improves expansion and persistence of chimeric antigen receptor-modified $\mathrm{T}$ cells in lymphoma patients. J Clin Invest. 2011;121:1822-6.

5. Jensen MC, Popplewell L, Cooper LJ, DiGiusto D, Kalos M, Ostberg JR, et al. Antitransgene rejection responses contribute to attenuated persistence of adoptively transferred CD20/CD19specific chimeric antigen receptor redirected $\mathrm{T}$ cells in humans. Biol Blood Marrow Transplant. 2010;16:1245-56.

6. Kochenderfer JN, Wilson WH, Janik JE, Dudley ME, StetlerStevenson M, Feldman SA, et al. Eradication of B-lineage cells and regression of lymphoma in a patient treated with autologous $\mathrm{T}$ cells genetically engineered to recognize CD19. Blood. 2010;116:4099-102.

7. Kochenderfer JN, Dudley ME, Feldman SA, Wilson WH, Spaner DE, Maric I, et al. B-cell depletion and remissions of malignancy along with cytokine-associated toxicity in a clinical trial of antiCD19 chimeric-antigen-receptor-transduced $\mathrm{T}$ cells. Blood. 2012;119:2709-20.

8. Kalos M, Levine BL, Porter DL, Katz S, Grupp SA, Bagg A, et al. $\mathrm{T}$ cells with chimeric antigen receptors have potent antitumor effects and can establish memory in patients with advanced leukemia. Sci Transl Med. 2011;3:95ra73.

9. Porter DL, Levine BL, Kalos M, Bagg A, June CH. Chimeric antigen receptor-modified $\mathrm{T}$ cells in chronic lymphoid leukemia. N Engl J Med. 2011;365:725-33.

10. Brentjens RJ, Riviere I, Park JH, Davila ML, Wang X, Stefanski J, et al. Safety and persistence of adoptively transferred autologous CD19-targeted T cells in patients with relapsed or chemotherapy refractory B-cell leukemias. Blood. 2011;118:4817-28.

11. Brentjens RJ, Davila ML, Riviere I, Park J, Wang X, Cowell LG, et al. CD19-targeted $\mathrm{T}$ cells rapidly induce molecular remissions in adults with chemotherapy-refractory acute lymphoblastic leukemia. Sci Transl Med. 2013;5:177ra138. 
12. Grupp SA, Kalos M, Barrett D, Aplenc R, Porter DL, Rheingold $\mathrm{SR}$, et al. Chimeric antigen receptor-modified $\mathrm{T}$ cells for acute lymphoid leukemia. N Engl J Med. 2013;368:1509-18.

13. Li YS, Hayakawa K, Hardy RR. The regulated expression of B lineage associated genes during $\mathrm{B}$ cell differentiation in bone marrow and fetal liver. J Exp Med. 1993;178:951-60.

14. Li YS, Wasserman R, Hayakawa K, Hardy RR. Identification of the earliest B lineage stage in mouse bone marrow. Immunity. 1996;5:527-35.

15. Kowolik CM, Topp MS, Gonzalez S, Pfeiffer T, Olivares S, Gonzalez N, et al. CD28 costimulation provided through a CD19specific chimeric antigen receptor enhances in vivo persistence and antitumor efficacy of adoptively transferred T cells. Cancer Res. 2006;66:10995-1004.

16. Loskog A, Giandomenico V, Rossig C, Pule M, Dotti G, Brenner MK. Addition of the CD28 signaling domain to chimeric T-cell receptors enhances chimeric $\mathrm{T}$-cell resistance to $\mathrm{T}$ regulatory cells. Leukemia. 2006;20:1819-28.

17. Brentjens RJ, Santos E, Nikhamin Y, Yeh R, Matsushita M, La Perle K, et al. Genetically targeted T cells eradicate systemic acute lymphoblastic leukemia xenografts. Clin Cancer Res. 2007;13:5426-35.

18. Pegram HJ, Lee JC, Hayman EG, Imperato GH, Tedder TF, Sadelain M, et al. Tumor-targeted T cells modified to secrete IL12 eradicate systemic tumors without need for prior conditioning. Blood. 2012;119:4133-41.

19. Cheadle EJ, Hawkins RE, Batha H, Rothwell DG, Ashton G, Gilham DE. Eradication of established B-cell lymphoma by CD19-specific murine $\mathrm{T}$ cells is dependent on host lymphopenic environment and can be mediated by CD4+ and CD8+ T Cells. J Immunother. 2009;32:207-18.

20. Cheadle EJ, Hawkins RE, Batha H, O'Neill AL, Dovedi SJ, Gilham DE. Natural expression of the CD19 antigen impacts the long-term engraftment but not antitumor activity of CD19-specific engineered T cells. J Immunol. 2010;184:1885-96.

21. Davila ML, Kloss CC, Gunset G, Sadelain M. CD19 CAR-targeted $\mathrm{T}$ cells induce long-term remission and $\mathrm{B}$ cell aplasia in an immunocompetent mouse model of B cell acute lymphoblastic leukemia. PLoS One. 2013;8:e61338.

22. Fielding AK, Richards SM, Chopra R, Lazarus HM, Litzow MR, Buck G, et al. Outcome of 609 adults after relapse of acute lymphoblastic leukemia (ALL); an MRC UKALL12/ECOG 2993 study. Blood. 2007;109:944-50.

23. O'Brien S, Schiller G, Lister J, Damon L, Goldberg S, Aulitzky W, et al. High-dose vincristine sulfate liposome injection for advanced, relapsed, and refractory adult Philadelphia chromosome-negative acute lymphoblastic leukemia. J Clin Oncol. 2013;31:676-83.

24. Topp MS, Gokbuget N, Zugmaier G, Degenhard E, Goebeler ME, Klinger M, et al. Long-term follow-up of hematologic relapsefree survival in a phase 2 study of blinatumomab in patients with MRD in B-lineage ALL. Blood. 2012;120:5185-7.

25. Porter DL, Grupp SA, Kalos M, Loren AW, Lledo L, Gilmore J, et al. Chimeric antigen receptor $\mathrm{T}$ cells directed against CD19 induce durable responses and transient cytokine release syndrome in relapsed, refractory CLL and ALL. ASH Annu Meet Abstr. 2012;120:717.

26. Park JH, Riviere I, Wang X, Stefanski J, He Q, Taylor C, et al. Impact of the conditioning chemotherapy on outcomes in adoptive $\mathrm{T}$ cell therapy: results from a phase I clinical trial of autologous CD19-targeted T cells for patients with relapsed CLL. ASH Annu Meet Abstr. 2012;120:1797.

27. Hackett PB, Largaespada DA, Cooper LJ. A transposon and transposase system for human application. Mol Ther. 2010;18:674-83.
28. Bonini C, Grez M, Traversari C, Ciceri F, Marktel S, Ferrari G, et al. Safety of retroviral gene marking with a truncated NGF receptor. Nat Med. 2003;9:367-9.

29. Zhong XS, Matsushita M, Plotkin J, Riviere I, Sadelain M. Chimeric antigen receptors combining 4-1BB and CD28 signaling domains augment PI3kinase/AKT/Bcl-XL activation and CD8+ T cell-mediated tumor eradication. Mol Ther. 2010;18:413-20.

30. Ciceri F, Bonini C, Stanghellini MT, Bondanza A, Traversari C, Salomoni M, et al. Infusion of suicide-gene-engineered donor lymphocytes after family haploidentical haemopoietic stem-cell transplantation for leukaemia (the TK007 trial): a non-randomised phase I-II study. Lancet Oncol. 2009;10:489-500.

31. Tey SK, Dotti G, Rooney CM, Heslop HE, Brenner MK. Inducible caspase 9 suicide gene to improve the safety of allodepleted $\mathrm{T}$ cells after haploidentical stem cell transplantation. Biol Blood Marrow Transplant. 2007;13:913-24.

32. Di Stasi A, Tey SK, Dotti G, Fujita Y, Kennedy-Nasser A, Martinez $\mathrm{C}$, et al. Inducible apoptosis as a safety switch for adoptive cell therapy. N Engl J Med. 2011;365:1673-83.

33. Giordano Attianese GM, Marin V, Hoyos V, Savoldo B, Pizzitola I, Tettamanti $S$, et al. In vitro and in vivo model of a novel immunotherapy approach for chronic lymphocytic leukemia by anti-CD23 chimeric antigen receptor. Blood. 2011;117:4736-45.

34. Trojan A, Schultze JL, Witzens M, Vonderheide RH, Ladetto M, Donovan JW, et al. Immunoglobulin framework-derived peptides function as cytotoxic T-cell epitopes commonly expressed in B-cell malignancies. Nat Med. 2000;6:667-72.

35. Vera J, Savoldo B, Vigouroux S, Biagi E, Pule M, Rossig C, et al. $\mathrm{T}$ lymphocytes redirected against the kappa light chain of human immunoglobulin efficiently kill mature B lymphocyte-derived malignant cells. Blood. 2006;108:3890-7.

36. Hudecek M, Schmitt TM, Baskar S, Lupo-Stanghellini MT, Nishida $\mathrm{T}$, Yamamoto TN, et al. The B-cell tumor-associated antigen ROR1 can be targeted with $\mathrm{T}$ cells modified to express a ROR1-specific chimeric antigen receptor. Blood. 2010;116:4532-41.

37. Siegel R, Naishadham D, Jemal A. Cancer statistics, 2013. CA Cancer J Clin. 2013;63:11-30.

38. Kumar SK, Rajkumar SV, Dispenzieri A, Lacy MQ, Hayman SR, Buadi FK, et al. Improved survival in multiple myeloma and the impact of novel therapies. Blood. 2008;111:2516-20.

39. Bellucci R, Alyea EP, Weller E, Chillemi A, Hochberg E, Wu CJ, et al. Immunologic effects of prophylactic donor lymphocyte infusion after allogeneic marrow transplantation for multiple myeloma. Blood. 2002;99:4610-7.

40. Lin P, Owens R, Tricot G, Wilson CS. Flow cytometric immunophenotypic analysis of 306 cases of multiple myeloma. Am J Clin Pathol. 2004;121:482-8.

41. Mihara K, Bhattacharyya J, Kitanaka A, Yanagihara K, Kubo T, Takei Y, et al. T-cell immunotherapy with a chimeric receptor against CD38 is effective in eliminating myeloma cells. Leukemia. 2012;26:365-7.

42. Benjamin R, Condomines M, Gunset G, Sadelain M. CD56 targeted chimeric antigen receptors for immunotherapy of multiple myeloma. In: Proceedings of the 103rd Annual Meeting of the American Association for Cancer Research. Cancer Res. 2012;72:3499.

43. Peinert S, Prince HM, Guru PM, Kershaw MH, Smyth MJ, Trapani JA, et al. Gene-modified $\mathrm{T}$ cells as immunotherapy for multiple myeloma and acute myeloid leukemia expressing the Lewis Y antigen. Gene Ther. 2010;17:678-86.

44. Carpenter RO, Evbuomwan MO, Pittaluga S, Rose JJ, Raffeld M, Yang S, et al. B-cell maturation antigen is a promising target for adoptive T-cell therapy of multiple myeloma. Clin Cancer Res. 2013;19:2048-60. 
45. Schuberth PC, Jakka G, Jensen SM, Wadle A, Gautschi F, Haley $\mathrm{D}$, et al. Effector memory and central memory NY-ESO-1-specific re-directed $\mathrm{T}$ cells for treatment of multiple myeloma. Gene Ther. 2013;20:386-95.

46. Marin V, Pizzitola I, Agostoni V, Attianese GM, Finney H, Lawson A, et al. Cytokine-induced killer cells for cell therapy of acute myeloid leukemia: improvement of their immune activity by expression of CD33-specific chimeric receptors. Haematologica. 2010;95:2144-52.

47. Dutour A, Marin V, Pizzitola I, Valsesia-Wittmann S, Lee D, Yvon E, et al. In vitro and in vivo antitumor effect of anti-CD33 chimeric receptor-expressing EBV-CTL against CD33 acute myeloid leukemia. Adv Hematol. 2012;2012:683065.

48. Tettamanti S, Marin V, Pizzitola I, Magnani CF, Giordano Attianese GM, Cribioli E, et al. Targeting of acute myeloid leukaemia by cytokine-induced killer cells redirected with a novel CD123-specific chimeric antigen receptor. Br J Haematol. 2013;161:389-401.

49. Ritchie DS, Neeson PJ, Khot A, Peinert S, Tai T, Tainton K, et al. Persistence and efficacy of second generation CAR $\mathrm{T}$ cell against the LeY antigen in acute myeloid leukemia. Mol Ther. 2013;21:2122-9.

50. Zhang S, Zhang HS, Cordon-Cardo C, Reuter VE, Singhal AK, Lloyd KO, et al. Selection of tumor antigens as targets for immune attack using immunohistochemistry: II Blood grouprelated antigens. Int J Cancer. 1997;73:50-6.

51. O'Hear C, Inaba H, Pounds S, Shi L, Dahl G, Bowman WP, et al. Gemtuzumab ozogamicin can reduce minimal residual disease in patients with childhood acute myeloid leukemia. Cancer 2013. 